Faculty of Mathematical Sciences

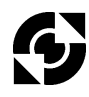

University of Twente

The Netherlands
P.O. Box 217

7500 AE Enschede

The Netherlands

Phone: +31-53-4893400

Fax: +31-53-4893114

Email: memo@math.utwente.nl

www.math.utwente. $\mathrm{nl} /$ publications

Memorandum No. 1668

The computational complexity of the

role assignment problem

J. Fiala ${ }^{1}$ and D. Paulusma

JANUARY, 2003

ISSN 0169-2690

\footnotetext{
${ }^{1}$ Charles University, Faculty of Mathematics and Physics, DIMATIA and Institute for Theoretical Computer Science (ITI), Malostranské nám. 2/25, 118 00, Prague, Czech Republic
} 


\title{
The computational complexity of the role assignment problem
}

\author{
Jiří Fiala ${ }^{* a}$ and Daniël Paulusma ${ }^{\dagger b}$
}

January 24, 2003

\author{
${ }^{a}$ Charles University, Faculty of Mathematics and Physics, \\ DIMATIA and Institute for Theoretical Computer Science (ITI) ${ }^{1}$, \\ Malostranské nám. 2/25, 118 00, Prague, Czech Republic. \\ fiala@kam.mff.cuni.cz \\ ${ }^{b}$ University of Twente, \\ Faculty of Electrical Engineering, Mathematics and Computer Science, \\ Department of Applied Mathematics, \\ P.O. Box 217, 7500 AE Enschede, The Netherlands. \\ d.paulusma@math.utwente.nl
}

\begin{abstract}
A graph $G$ is $R$-role assignable if there is a locally surjective homomorphism from $G$ to $R$, i.e. a vertex mapping $r: V_{G} \rightarrow V_{R}$, such that the neighborhood relation is preserved: $r\left(N_{G}(u)\right)=N_{R}(r(u))$. Kristiansen and Telle conjectured that the decision problem whether such a mapping exists is an NP-complete problem for any connected graph $R$ on at least three vertices. In this paper we prove the conjecture and show further corollaries for disconnected graphs and related problems.
\end{abstract}

Keywords: computational complexity, graph homomorphism, role assignment

2002 Mathematics Subject Classification: 05C15, 03D15.

\section{Introduction}

Given two graphs, say $G$ and $R$, an $R$-role assignment for $G$ is a vertex mapping $r: V_{G} \rightarrow V_{R}$, such that the neighborhood relation is maintained, i.e. all roles of the image of a vertex appear on the vertex's neighborhood. Such a condition can be formally expressed as

$$
\text { for all } u \in V_{G}: r\left(N_{G}(u)\right)=N_{R}(r(u)) \text {, }
$$

\footnotetext{
*This author was partially supported by research grant GAUK 158/99.

$\dagger$ This author was partially supported by NWO grant R 61-507 and by Czech research grant GAČR 201/99/0242 during his stay at DIMATIA center in Prague.

${ }^{1}$ Supported by the Ministry of Education of the Czech Republic as project LN00A056.
} 
where $N(u)$ denotes the set of neighbors of $u$ in the corresponding graph.

Such assignments have been introduced by Everett and Borgatti [6], who called them role colorings. They originated in the theory of social behavior. The graph $R$, i.e. the role graph, models roles and their relationships, and for a given society we can ask whether its individuals can be assigned roles such that the relationships are preserved: Each person playing a particular role has among its neighbors exactly all necessary roles as they are prescribed by the model.

From the computational complexity point of view it is interesting to know whether it is possible to decide quickly (i.e. in polynomial time) whether such assignment exists. This problem was considered by Roberts and Sheng [15], who focus on a more generalized problem called the 2-role assignment problem. If both graphs $G$ and $R$ are part of the input, the problem is NP-complete already for $R=K_{3}$ [12].

In order to make a more precise study we consider a class of $R$-role assignment problems, $\mathrm{RA}(R)$, parameterized by the role graph $R$. Here the instance is formed only by the graph $G$, and we ask whether an $R$-role assignment of $G$ exists.

The complexity study of this class of problems is closely related to a similar approach for locally constrained graph homomorphism problems [9]. A graph homomorphism from $G$ to $H$ is a vertex mapping $f: V_{G} \rightarrow V_{H}$ satisfying the property that whenever an edge $(u, v)$ appears in $E_{G}$, then $(f(u), f(v))$ belongs to $E_{H}$ as well.

The adjective "locally constrained" expresses the condition that the mapping $f$ restricted to the neighborhood of any vertex $u$ must satisfy further properties. (See $[14,7]$ for a general model of such conditions.)

It may be required to be locally

- bijective, then the mapping is called a full cover of $H$, and the corresponding decision problem is called $H$-Cover $[1,13]$,

- injective, then it is called a partial cover of $H$, and the problem $H$ PCover $[8,9]$,

- surjective, then we get a locally surjective cover of $H$, and decision problem $H$-Colordomination [14].

All these problems are parameterized by a fixed graph $H$, and the instance is formed only by a graph $G$. The question is whether an appropriate graph homomorphism from $G$ to $H$ exists. Observe that the definition of a locally surjective cover is equivalent with the definition of an $R$-role assignment for $R=H$.

Full covers have important applications, for example in distributed computing [5], in recognizing graphs by networks of processors [2, 3], or in constructing highly transitive regular graphs [4]. Similarly partial covers are used in distance constrained labelings of graphs [10]. 
Even if the first attempt to get some results on the computational complexity for the class of $H$-Cover problems was made a decade ago in [1], it is not fully classified yet neither for $H$-PCover nor for $H$-Colordomination (RA $(H)$ ) problems. However, several partial results are known. For example, if the $H$ Cover problem is NP-complete, then the corresponding $H$-PCover [9] and $H$-Colordomination problems [14] are NP-complete as well. Moreover, the $H$-Cover problem is known to be NP-complete for all $k$-regular graphs $H$ of valency $k \geq 3$ [9], and the NP-hardness hence propagates for partial and locally surjective covers of such graphs as well.

The $H$-COLORDOMINATION problem was proven to be NP-complete for paths, cycles and stars in [14]. It was conjectured there that for simple connected graphs the $H$-Colordomination problem is NP-complete if and only if $H$ has at least three vertices. Our main theorem proves this conjecture.

The paper is organized as follows. The next section provides necessary definitions and basic observations. The third section provides technical lemmas used in the main construction which is showed in the fourth section. The fifth section describes the complexity of the role assignment problem for disconnected role graphs. We finally apply the main theorem to prove NP-completeness for a generalized $k$-role assignment problem [15] in the sixth section.

\section{Preliminaries}

Through the paper we use terminology stemming from the role assignment problems.

We consider simple graphs, denoted by $G=\left(V_{G}, E_{G}\right)$, where $V_{G}$ is a finite vertex set of vertices and $E_{G}$ is a set of unordered pairs of vertices, called edges. For a vertex $u \in V_{G}$ we denote its neighborhood, i.e. the set of adjacent vertices, by $N_{G}(u)=\left\{v \mid(u, v) \in E_{G}\right\}$.

The degree $\operatorname{deg}_{G}(u)$ of a vertex $u$ is the number of edges incident with it, or equivalently the size of its neighborhood. The symbol $\delta(G)$ is the minimum degree among all vertices of $G$.

A graph $G$ is called connected if for every pair of distinct vertices $u$ and $v$, there exists a path connecting $u$ and $v$, i.e. a sequence of distinct vertices starting by $u$ and ending by $v$ where each pair of consecutive vertices forms an edge of $G$. The length of the path is the number of its edges.

A graph that is not connected is called disconnected. Each maximal connected subgraph of a graph is called a component. A vertex whose removal causes a component of a graph to become disconnected is called a cutvertex. We say that a cutvertex $u$ separates vertex $v$ from $w$ in $G$ if $v, w$ belong to different components of $G \backslash u$.

Two graphs $G$ and $\tilde{G}$ are called isomorphic, denoted by $G \simeq \tilde{G}$, if there exists a one-to-one mapping $f$ of vertices of $G$ onto vertices of $\tilde{G}$ such that $(u, v) \in E_{G}$ if and only if $(f(u), f(v)) \in E_{\tilde{G}}$.

In the sequel the symbol $G$ denotes the instance graph and $R$ the so-called role graph. 
Definition We say that $G$ is $R$-role assignable if a mapping $r: V_{G} \rightarrow V_{R}$ exists satisfying:

$$
\text { for all } u \in V_{G}: r\left(N_{G}(u)\right)=N_{R}(r(u)) \text {, }
$$

where we use the notation $r(S)=\bigcup_{u \in S} r(u)$ for a set of vertices $S \subseteq V_{G}$. The function $r$ is called an $R$-role assignment of $G$.

The goal of this paper is a full characterization of the computational complexity for the following class of problems:

$R$-Role Assignment $(\mathrm{RA}(R))$

Instance: A graph $G$.

Question: Does the graph $G$ allow an $R$-role assignment?

We continue with some observations that we use later in the paper. We note first that role assignments are closed under composition:

Observation 2.1 If $G$ is $S$-role assignable and $S$ is $R$-role assignable, then $G$ is $R$-role assignable.

Proof: Let $s: V_{G} \rightarrow V_{S}$ be an $S$-role assignment for $G$ and $r: V_{S} \rightarrow V_{R}$ be an $R$-role assignment for $S$. Then $t: V_{G} \rightarrow V_{R}$ defined by $t(u)=r(s(u))$ for all $u \in V_{G}$ is an $R$-role assignment for $G$.

Observation 2.2 If $G$ is $R$-role assignable, then $\operatorname{deg}_{G}(u) \geq \operatorname{deg}_{R}(r(u))$ for all vertices $u \in V_{G}$.

Proof: $\operatorname{deg}_{G}(u)=\left|N_{G}(u)\right| \geq\left|r\left(N_{G}(u)\right)\right|=\left|N_{R}(r(u))\right|=\operatorname{deg}_{R}(r(u))$.

From this we easily derive that $\delta(G) \geq \delta(R)$, and moreover:

Lemma 2.3 If $G$ is $R$-role assignable and $u$ is a vertex of $G$ with $\operatorname{deg}_{G}(u)=$ $\delta(R)$, then $\operatorname{deg}_{R}(r(u))=\delta(R)$ and $r$ restricted to $N_{G}(u)$ is an isomorphism between $N_{G}(u)$ and $N_{R}(r(u))$.

Lemma 2.4 Let $G$ be $R$-role assignable and $x, y$ be vertices of $R$ connected by a path $P_{R}$. Then for each $u$ with $r(u)=x$ a vertex $v \in V_{G}$ and a path $P_{G}$ connecting $u$ and $v$ exist, such that $r$ restricted to $P_{G}$ is an isomorphism between $P_{G}$ and $P_{R}$.

Proof: We prove the statement by induction on the length of the path $P_{R}$. If $x$ and $y$ are adjacent, then the vertex $u$ has a neighbor $v$ mapping onto $y$, by the definition of the $R$-role assignment $r$.

Now assume that the path $P_{R}$ is of length $k \geq 2$, and that the hypothesis is valid for all paths of length at most $k-1$. Denote by $y^{\prime}$ the predecessor of $y$ in $P_{R}$ and by $P_{R}^{\prime}$ the truncation of $P_{R}$ by the last edge, i.e. the path of length $k-1$ connecting $x$ and $y^{\prime}$. By the induction hypothesis $G$ contains a vertex $v^{\prime}$ and a path $P_{G}^{\prime}$ such that $P_{G}^{\prime} \simeq P_{R}^{\prime}$ under $r$. Then it is easy to find a neighbor $v$ of $v^{\prime}$ satisfying $r(v)=y$ and tack it to $P_{G}^{\prime}$ to get the desired path $P_{G}$.

We get immediately the following claims: 
Observation 2.5 If $G$ is $R$-role assignable and $R$ is connected, then each vertex $v \in V_{R}$ appears as a role for some vertex $u \in V_{G}$.

If $G \simeq R$ then $G$ is $R$-role assignable, because every isomorphism satisfies the condition of the role assignment. Due to the previous observation we have:

Observation 2.6 Let $R$ be a connected role graph. If $G$ is $R$-role assignable and $\left|V_{G}\right|=\left|V_{R}\right|$, then $G \simeq R$.

Lemma 2.7 Let $G$ be $R$-role assignable, $u \in V_{G}$ be a vertex of role $x$, and $z, y \in V_{R}$ be some other roles. If in $G$ each path connecting u to a vertex of role $y$ contains a vertex of role $z$, then the vertex $z$ is a cutvertex in $R$.

Proof: Since vertices of roles $x$ and $y$ are connected by a path in $G$, there exists a path in $R$ connecting $x$ to $y$. Moreover if $z$ were not a cutvertex, then we can find such a path avoiding the role $z$. But then by Lemma 2.4 we can find a path in $G$ from $u$ to some vertex of role $y$ avoiding any vertex of role $z$.

Lemma 2.8 Let $u$ be a cutvertex of $G$ and $C$ be a component of $G \backslash u$. Then the roles of $V_{C}$ form a connected subgraph of $R$.

Proof: By the definition, whenever $(u, v) \in E_{G}$ then $(r(u), r(v)) \in E_{R}$, in other words, every role assignment is also a graph homomorphism. In any homomorphism the image of a connected graph is connected.

Lemma 2.9 Let $G$ be $R$-role assignable, $u, u^{\prime}$ be vertices of $G$ such that $N_{G}(u) \subseteq$ $N_{G}\left(u^{\prime}\right)$, and $\operatorname{deg}_{G}(u)=\delta(R)$. If all vertices of minimum degree in $R$ are cutvertices then $r(u)=r\left(u^{\prime}\right)$.

Proof: We denote $z=r(u)$. Since $\operatorname{deg}_{R}(z) \leq \operatorname{deg}_{G}(u)=\delta(R)$ we get that $z$ is a vertex of minimum degree, and by our assumptions it is also a cutvertex in $R$. Let $x, y$ be two of its neighbors that are separated by $z$ and let $v, w \in N_{G}(u)$ be their preimages. (Their uniqueness is even guaranteed by Lemma 2.3.) The image of the path $v, u^{\prime}, w$ is connected, hence it contains the vertex $z$ as the role of $u^{\prime}$.

\section{Gadgets}

\subsection{Graphs with two role assignments}

For the garbage collection in our NP-completeness proof we need to construct a graph that allows two different role assignments:

Lemma 3.1 Let $R$ be a role graph. Then a graph $H$ exists that has two $R$-role assignments $r_{1}$ and $r_{2}$, such that for any two roles $v$ and $w$, a vertex $u$ exists in $H$ with $r_{1}(u)=v$, and $r_{2}(u)=w$. Moreover, $H$ can be constructed in time being polynomial with respect to the size of $R$. 
Proof: Take $H$ as the Cartesian product $R \times R$, defined by the vertex set $V_{H}=V_{R} \times V_{R}$, and edges $((a, b),(c, d)) \in E_{H}$ if and only if $(a, c),(b, d) \in E_{R}$.

The projections $r_{1}:(a, b) \rightarrow a$ and $r_{2}:(a, b) \rightarrow b$ are valid $R$-role assignments, and the vertex $u=(v, w)$ satisfies the statement of the Lemma.

Note that for our purposes, it is possible for any two roles $v, w$ to construct a connected $H$ with two role assignments - it is enough to select the component of $R \times R$ containing the vertex $u=(v, w)$.

\subsection{Glued subgraphs}

Definition We say that a graph $\tilde{R}$ is glued in a graph $G$ by a vertex $\tilde{v}$, if $G$ can be obtained from $\tilde{R}$ and some other graph $G^{\prime}$ by identifying a vertex $x \in V_{G^{\prime}}$ with the vertex $\tilde{v}$.

See Fig. 1 for a more intuitive picture of such a glued graph.

As a convention we use letters $x, y, z$ to denote roles, while $u$ is reserved for vertices of the instance. The symbols $v, w$ stand for roles, while $\tilde{v}$ or $w^{\prime}$ are vertices of the instance graph isomorphic to $v, w$.

Lemma 3.2 Let $R$ be a connected role graph. Let $G$ be an $R$-role assignable graph and $\tilde{R}$ be glued in $G$ by a vertex $\tilde{v}$, where $\tilde{R}$ is isomorphic to $R$ and $v$, the isomorphic copy of $\tilde{v}$ in $\tilde{R}$, is not a cutvertex of $R$. Then $r\left(V_{\tilde{R}}\right)=V_{R}$.

Proof: We use induction on the number of vertices.

If $R$ consists of only one vertex, then $G$ has no edges. Similarly if $R$ consists of only two vertices, then $G$ must be bipartite without isolated vertices and the statement is valid directly.

For graphs $R$ on at least three vertices we prove the statement by contradiction. Suppose a role $z \in V_{R} \backslash r\left(V_{\tilde{R}}\right)$ exists.

Claim 1: The role $v^{\prime}$ of the vertex $\tilde{v}$ is a cutvertex of $R$ and does not appear as a role of any other vertex of $\tilde{R}$.

Select any vertex $u$ of $\tilde{R}$ other from $\tilde{v}$. An arbitrary path from $u$ to a vertex with the role $z$ uses $v^{\prime}$, hence $v^{\prime}$ is a cutvertex by Lemma 2.7. If $r(u)=v^{\prime}$ then by Lemma 2.4 it would be possible to select a path using distinct nodes. This contradicts the fact that $v^{\prime}$ is used on the path at least twice.

Based on this claim we can explore the structure of $R$. Denote by $S$ the subgraph of $R$ induced by $r\left(V_{\tilde{R}}\right)$. Since $v$ is not a cutvertex, the graph $\tilde{R} \backslash \tilde{v}$ is connected. Lemma 2.8 shows that $S \backslash v^{\prime}$ is connected as well. Hence the vertex $v^{\prime}$ is not a cutvertex in $S$, but it is a cutvertex of $R$.

Claim 2: The mapping $s: V_{\tilde{R}} \rightarrow V_{S}$ defined by $s(u)=r(u)$ for all $u \in V_{\tilde{R}}$ is an $S$-role assignment for $\tilde{R}$.

For all $u \in V_{\tilde{R}} \backslash \tilde{v}$ we get

$$
s\left(N_{\tilde{R}}(u)\right)=r\left(N_{G}(u)\right)=N_{R}(r(u))=N_{S}(s(u)) .
$$

We have to show the same statement also for $\tilde{v}$. Firstly we get that $s\left(N_{\tilde{R}}(\tilde{v})\right)=$ $r\left(N_{\tilde{R}}(\tilde{v})\right) \subseteq N_{R}\left(v^{\prime}\right) \cap V_{S}=N_{S}\left(v^{\prime}\right)=N_{S}(s(\tilde{v}))$. The inclusion "ᄃ" is in fact 


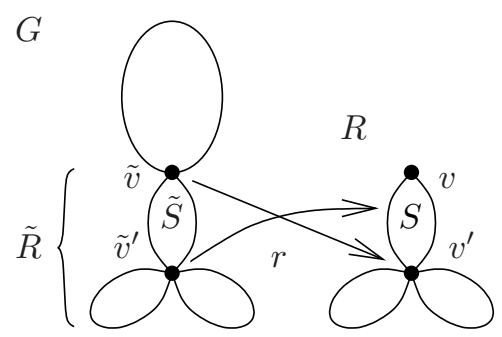

Figure 1: A role assignment of a glued subgraph

equality "=". Assume that some role $z \in N_{R}\left(v^{\prime}\right) \cap V_{S}$ does not appear among roles of $N_{\tilde{R}}(\tilde{v})$ under the assignment $s$. Since $z \in V_{S}$, a vertex $u$ of $\tilde{R}$ exists such that $s(u)=z$. Then $u \neq \tilde{v}$ and it has a neighbor $u^{*} \neq \tilde{v}$ with role $v^{\prime}$, a contradiction with Claim 1.

Let $\tilde{S}$ be the subgraph of $\tilde{R}$ corresponding to $S$. Since the role graph $S$ has less vertices than $R$, and the mapping $s$ is a proper $S$-role assignment, we get by the induction hypothesis that

$$
r\left(V_{\tilde{S}}\right)=s\left(V_{\tilde{S}}\right)=V_{S} .
$$

We focus our attention on other properties of cutvertices:

Claim 3: If $u$ is a cutvertex of $\tilde{R}$ then $r(u)$ is a cutvertex of $R$.

To see this consider in $\tilde{R}$ a vertex $u^{*}$ separated from $\tilde{v}$ by $u$. According to Claim 1 the role $v^{\prime}$ appears only once on $\tilde{R}$, namely as the image of the vertex $\tilde{v}$. Then every path in $G$ from $u^{*}$ to a vertex of role $v^{\prime}$ uses the vertex $u$. By Lemma 2.7 the role $r(u)$ is a cutvertex in $R$.

We formulate a similar statement for cutvertices in $\tilde{S}$.

Claim 4: If $u$ is a cutvertex of $\tilde{S}$ then $r(u)$ is a cutvertex of $S$.

Because the graph $S$ is separated from the rest of the graph $R$ by the vertex $v^{\prime}$, it follows that for each cutvertex of $S$, its isomorphic copy is not only a cutvertex in $\tilde{S}$ but also in $\tilde{R}$. Then, according to Claim 3, the cutvertices of $\tilde{S}$ map onto cutvertices of $R$, which are not equal to $v^{\prime}$ due to Claim 1 . Hence they must be mapped on cutvertices of $S$.

We are ready to conclude our proof. Since $r\left(V_{\tilde{S}}\right)=V_{S}$ and $\tilde{S}$ is isomorphic to $S$, the role assignment $r$ acts as a bijection between $V_{\tilde{S}}$ and $V_{S}$. This fact together with Claim 4 implies that $r$ maps all non-cutvertices of $\tilde{S}$ on noncutvertices of $S$.

The vertex $\tilde{v}^{\prime}$, i.e the isomorphic copy of $v^{\prime}$, maps on a cutvertex in $S$, because it is a cutvertex in $\tilde{R}$. However, it is not a cutvertex in $\tilde{S}$ (see Fig. 1).

Lemma 3.3 Let $R$ be a connected role graph. Let $G$ be an $R$-role assignable graph and $\tilde{R}$ be glued in $G$ by a vertex $\tilde{v}$, where $\tilde{R}$ is isomorphic to $R$ and $v$, the isomorphic copy of $\tilde{v}$ in $\tilde{R}$, is not a cutvertex of $R$. Then an $R$-role assignment $r$ exists such that $r(\tilde{w})=w$ for every $w \in V_{R}$. 
Proof: By Lemma 3.2 every $R$-role assignment $s: V_{G} \rightarrow V_{R}$ acts as a bijection when restricted to $\tilde{R}$. This mapping defines an isomorphism $\pi: V_{R} \rightarrow V_{R}$ by

$$
\pi(x)=w \quad \Leftrightarrow \quad s(\tilde{w})=x
$$

which is also an $R$-role assignment of $R$. We have already observed that the composition of two role assignments is a role assignment. Then $r=\pi \circ s$ is an $R$-role assignment of $G$ satisfying $r(\tilde{w})=\pi(s(\tilde{w}))=w$.

\section{The main result}

Now we are ready to prove the conjecture of Kristiansen and Telle [14]:

Theorem 1 Let $R$ be a connected role graph. Then the $R$-role assignment problem is polynomially solvable if $\left|V_{R}\right| \leq 2$ and it is NP-complete if $\left|V_{R}\right| \geq 3$.

Proof: First we show that $\mathrm{RA}(R)$ is polynomially solvable for $\left|V_{R}\right| \leq 2$.

- $\left|V_{R}\right|=1$. Clearly, a graph $G$ is $R$-role assignable if and only if $G$ contains only isolated vertices.

- $\left|V_{R}\right|=2$. Clearly, a graph $G$ is $R$-role assignable if and only if $G$ is a bipartite graph that does not contain any isolated vertices.

Now let $\left|V_{R}\right| \geq 3$. Since we can guess a mapping $r: V_{G} \rightarrow V_{R}$ and check in polynomial time if $r$ is an $R$-role assignment, the problem $\mathrm{RA}(R)$ is a member of NP. We prove NP-completeness by reduction from hypergraph 2-colorability. This is a well-known NP-complete problem (cf. [11]).

HyPERGRAPH 2-COLORABILITy (H2C)

Instance: A set $Q=\left\{q_{1}, \ldots, q_{m}\right\}$ and a set $\mathcal{S}=\left\{S_{1}, \ldots, S_{n}\right\}$ with $S_{j} \subseteq X$ for $1 \leq j \leq n$.

Question: Is there a 2-coloring of $(Q, \mathcal{S})$, i.e., a partition of $Q$ into $Q_{1} \cup Q_{2}$ such that $Q_{1} \cap S_{j} \neq \emptyset$ and $Q_{2} \cap S_{j} \neq \emptyset$ for $1 \leq j \leq n$ ?

With such a hypergraph we associate its incidence graph $I$, which is a bipartite graph on $Q \cup \mathcal{S}$, where $(q, S)$ forms an edge if and only if $q \in S$.

To prove the theorem we choose a vertex $v \in V_{R}$ of minimum degree. Because we cannot apply Lemma 3.3 if $v$ is a cutvertex, we have to distinguish between the case, in which all vertices of minimum degree are cutvertices, and the case, in which a non-cutvertex of minimum degree exists.

Assume first that the vertex $v$ is a vertex of minimum degree that is not a cutvertex. Denote the neighbors of $v$ by $N_{R}(v)=\left\{w_{1}, \ldots, w_{p}\right\}$ and also the second common neighborhood as $M_{R}(v)=\bigcap_{u \in N_{R}(v)} N_{R}(u)=\left\{v, v_{2}, \ldots, v_{l}\right\}$. See Fig. 2 for a drawing of a possible situation.

We distinguish four cases according to possible values of $p$ and $l$ :

Case 1: $p=1, l=1$. Then $R=K_{2}$ and we have already discussed this case above. 


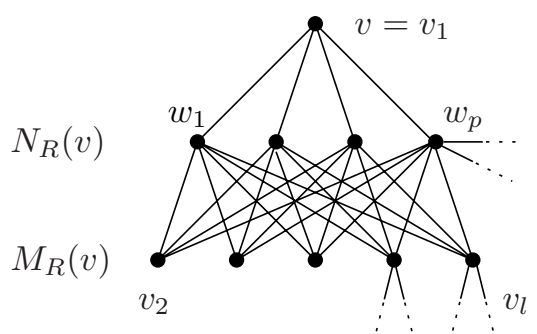

Figure 2: Neighborhood of a vertex $v$ in $R$.

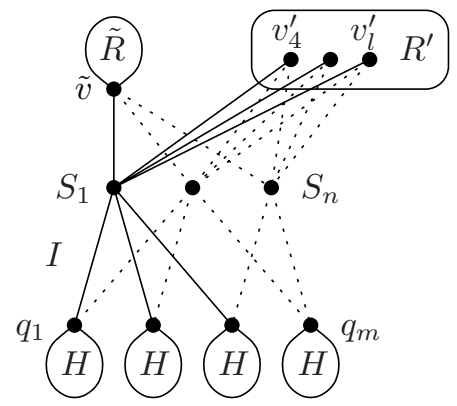

Figure 3: Construction of the graph $G$ in Case 2.

Case 2: $p=1, l \geq 3$. We extend the incidence graph $I$ as follows: According to Lemma 3.1 we construct a graph $H$ for which two role assignments exist mapping a particular vertex $u$ to $v_{2}$ and $v_{3}$. We form an instance $G$ as the union of the graph $I$ and $m$ disjoint copies of the graph $H$, where the vertex $u$ of the $i$-th copy is identified with the vertex $q_{i}$ of $I$. Finally we insert into $G$ two extra copies $\tilde{R}, R^{\prime}$ of the role graph $R$ and add the following edges (cf. Fig 3):

- $\left(\tilde{v}, S_{j}\right)$ for all $S_{j} \in \mathcal{S}$,

- $\left(v_{k}^{\prime}, S_{j}\right)$ for all $S_{j} \in \mathcal{S}$ and all $4 \leq k \leq l$ (this set may be empty).

We show that the graph $G$ formed in this way allows an $R$-role assignment if and only if $(Q, \mathcal{S})$ is 2-colorable.

Assume first that $G$ is $R$-role assignable. Then according to Lemma 3.3 we assume that the vertex $\tilde{v}$ is assigned role $v$ and all vertices $S_{j}$ are mapped to role $w_{1}$. Since their neighborhoods are saturated by common $l-3$ roles on $v_{4}^{\prime}, \ldots, v_{l}^{\prime}$, at least two distinct roles $v_{a}, v_{b} \in M_{R}(v) \backslash r\left(\left\{v_{4}^{\prime}, \ldots, v_{l}^{\prime}\right\}\right)$ exist that are used on some neighbors of each $S_{j}$ in the set $\mathcal{S}$.

The partition $Q_{1}=\left\{q_{i} \mid r\left(q_{i}\right)=v_{a}\right\}$ and $Q_{2}=Q \backslash Q_{1} \supseteq\left\{q_{i} \mid r\left(q_{i}\right)=v_{b}\right\}$ is the desired 2-coloring of $(Q, \mathcal{S})$.

In the opposite direction, any 2-coloring $Q_{1}, Q_{2}$ can be transformed into an $R$-role assignment $r$ of $G$ by letting $r\left(q_{i}\right)=v_{a}$ if $q_{i} \in Q_{a}$ for $a=1,2$ and by 


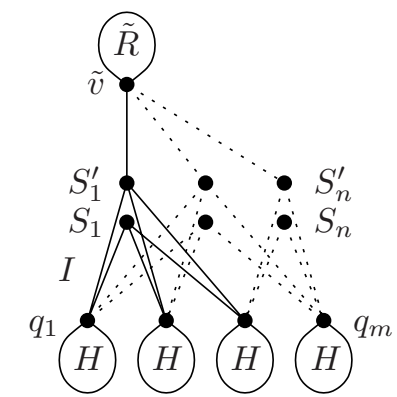

Figure 4: Construction of the graph $G$ in Case 3 .

further extension according to the two projections of the graph $H$ and graph isomorphisms $\tilde{R} \rightarrow R, R^{\prime} \rightarrow R$.

Case 3: $p=1, l=2$. The case when $R$ is isomorphic to the path $P_{4}$ was already shown to be NP-complete in [14]. If $R$ is not isomorphic to a path on four vertices but $v_{2}$ is incident with a vertex $v^{*}$ of degree one, then we can reduce this case to the previous case $(p=1, l \geq 3)$ by selecting $v^{*}$ as the non-cutvertex of minimum degree. So without loss of generality we may assume that $v_{2}$ is not incident with a vertex of degree one.

We construct $G$ from $I$ as follows. First we insert $n$ new vertices $S_{1}^{\prime}, \ldots, S_{n}^{\prime}$ and a copy $\tilde{R}$ of the role graph $R$. We identify each $q_{i}$ with the vertex $u$ of an extra copy of the graph $H$ as in the previous case, but here $H$ is constructed such that $u$ can be assigned $v$ or $v_{2}$.

These parts are linked as follows (cf. Fig. 4):

- $\left(\tilde{v}, S_{j}^{\prime}\right) \in E_{G}$ for all $j \in\{1, \ldots, n\}$,

- $\left(q_{i}, S_{j}^{\prime}\right) \in E_{G}$ if and only if $\left(q_{i}, S_{j}\right) \in E_{I}$.

If $G$ is $R$-role assignable, then without loss of generality we may assume that $\tilde{v}$ has role $v$. Then all $S_{j}^{\prime}$ have role $w_{1}$ since $w_{1}$ is the only neighbor of $v$. The roles of all $q_{i}$ hence belong to $N_{R}\left(w_{1}\right)=\left\{v, v_{2}\right\}$. Each $S_{j}^{\prime}$ requires the role $v_{2}$ to be present among its neighbors in $Q$. Moreover, if all neighbors of some $S_{j}^{\prime}$ in $Q$ are assigned the role $v_{2}$, we get that $S_{j}$ must be mapped to a neighbor of $v_{2}$ that is a leaf, but this is in contradiction with our assumptions. We conclude that each $S_{j}$ is mapped to $w_{1}$. Hence both roles $v, v_{2}$ appear on its neighborhood and the partition $Q_{1}=\left\{q_{i} \mid r\left(q_{i}\right)=v\right\}$ and $Q_{2}=\left\{q_{i} \mid r\left(q_{i}\right)=v_{2}\right\}$ is a 2-coloring of $(Q, \mathcal{S})$.

In the opposite direction, an $R$-role assignment of $G$ can be constructed from a 2-coloring of $(Q, \mathcal{S})$ in a straightforward way as in the previous case.

Case 4: $p \geq 2$. As above we first build the graph $H$ which allows two $R$-role assignments mapping a vertex $u$ either to $w_{1}$ or to $w_{2}$.

The graph $G$ consists of the graph $I$, where each $q_{i}$ is unified with the vertex $u$ of an extra copy of $H$. We further include two copies of $R$ denoted by $\tilde{R}$ and $R^{\prime}$. Finally we extend the set of edges by (cf. Fig. 5): 


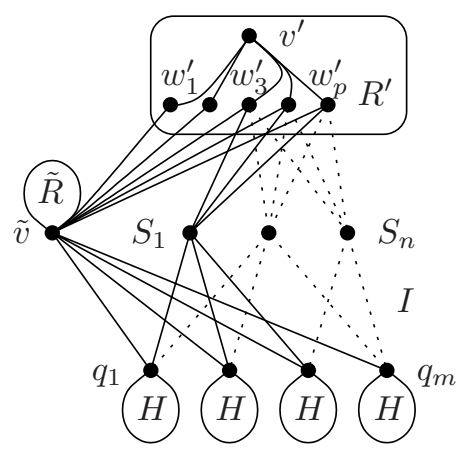

Figure 5: Construction of the graph $G$ in Case 4.

- $\left(\tilde{v}, q_{i}\right)$ for all $q_{i} \in Q$,

- $\left(\tilde{v}, w_{k}^{\prime}\right)$ for all $1 \leq k \leq p$,

- $\left(S_{j}, w_{k}^{\prime}\right)$ for all $3 \leq k \leq p$ (this set may be empty).

If an $R$-role assignment exists, then we assume that $r(\tilde{v})=v$. For each $S_{j}$ we have $N_{G}\left(S_{j}\right) \subseteq N_{G}(\tilde{v})$. So we know that $S_{j}$ is assigned some role $v_{i}$ for which $N_{R}\left(v_{i}\right)=N_{R}(v)$. However only $p-2$ roles appear on vertices $w_{3}^{\prime}, \ldots, w_{p}^{\prime}$, so two distinct roles $w_{a}$ and $w_{b}$ are used on none of $w_{3}^{\prime}, \ldots, w_{p}^{\prime}$. Then we define a 2-coloring of $(Q, \mathcal{S})$ by selecting $Q_{1}=\left\{q_{i} \mid r\left(q_{i}\right)=w_{a}\right\}$ and $Q_{2}=Q \backslash Q_{1} \supseteq$ $\left\{q_{i} \mid r\left(q_{i}\right)=w_{b}\right\}$.

An $R$-role assignment can be derived from a 2-coloring of $(Q, \mathcal{S})$ as in the previous cases.

Finally, we return to the situation when all vertices of minimum degree in $R$ are cutvertices. (Observe, that $\delta(R) \geq 2$ since vertices of degree one are not cutvertices.)

We construct the graph $G$ as in Case 4 above (cf. Fig. 5). The argumentation goes in the same manner: Since $N_{G}\left(v^{\prime}\right) \subseteq N_{G}(\tilde{v})$, we get by Lemma 2.9 that $\tilde{v}$ is mapped to a role of minimum degree. For each $S_{j}$ we have $N_{G}\left(S_{j}\right) \subseteq N_{G}(\tilde{v})$. So we know that $S_{j}$ is assigned a role that has the same neighbors in $R$ as role $r(\tilde{v})$. Each $S_{j}$ then lacks two roles $w_{a}, w_{b}$ that do not appear on $w_{3}^{\prime}, \ldots, w_{p}^{\prime}$. Hence we can define a valid 2-coloring of $(Q, \mathcal{S})$ according to the appearance of roles $w_{a}$ and $w_{b}$ on the set $Q$.

Observe that all graphs $G$ involved in our constructions were connected, even if the incidence graph $I$ was not connected.

\section{Disconnected Role Graphs}

Up to now we have only considered role graphs that were connected. Due to this property we could easily derive that all roles appear as the image of the 
vertex in the instance graph (cf. Observation 2.5). We now focus our attention to the case of disconnected role graphs. Suppose $R$ is a role graph with set of components $\mathcal{C}=\left\{C_{1}, \ldots C_{m}\right\}$. We order the components such that the latter have a higher number of vertices. (Formally, for all $i \leq j:\left|V_{C_{i}}\right| \leq\left|V_{C_{j}}\right|$.)

Note that the identity mapping $\pi: V_{C_{1}} \rightarrow V_{R}$ preserves the local constraint for role assignment, but Observation 2.5 is no longer valid here (take $G \simeq C_{1}$ ). Our argument guarantees that a locally surjective cover is globally surjective only for connected role graphs. Within some social network models it is natural to demand that all roles appear on the vertices of the instance graph. We show below that the computational complexity of the role assignment problem for disconnected role graphs depends whether such a property $r\left(V_{G}\right)=V_{R}$ is required or not.

We call an $R$-role assignment $r: V_{G} \rightarrow V_{R}$ a globally $R$-role assignment for $G$ if $r$ is an $R$-role assignment and $r\left(V_{G}\right)=V_{R}$ holds. Our generalized role assignment problem can now be formulated as

Global $R$-Role Assignment $(\operatorname{GRA}(R))$

Instance: A graph $G$.

Question: Is $G$ globally $R$-role assignable?

With respect to the computational complexity we obtain the following result.

Theorem 2 Let $R$ be a disconnected role graph. Then the $\operatorname{GRA}(R)$ problem is polynomially solvable if all components have at most two vertices and it is NP-complete otherwise.

Proof: Clearly the GRA $(R)$ problem belongs to NP. For connected role graphs the statement immediately follows from Theorem 1.

Suppose $R$ has $m \geq 2$ components ordered as shown above. If all components consist of only one vertex, then a graph $G$ is $R$-role assignable if and only if $G$ is a collection of at least $m$ isolated vertices. Suppose $R$ consists of $k$ isolated vertices and $m-k$ isolated edges. Then a graph $G$ is $R$-role assignable if and only if $G$ contains at least $k$ isolated vertices and at least $m-k$ bipartite components, each on at least two vertices.

Now suppose $\left|V_{C_{m}}\right| \geq 3$. We prove NP-completeness by reduction from $\operatorname{RA}\left(C_{m}\right)$. Without loss of generality we assume that the instance graph $G$ for the $\operatorname{RA}\left(C_{m}\right)$ problem is connected. Let $G^{\prime}$ be the graph with components $G, \tilde{C}_{1}, \ldots, \tilde{C}_{m-1}$, where $\tilde{C}_{i}$ is isomorphic to $C_{i}$ for $1 \leq i \leq m-1$. It is straightforward to see that $G^{\prime}$ is $R$-role assignable if $G$ is $C_{m}$-role assignable.

Now assume that $G^{\prime}$ is $R$-role assignable. Observe that both $G^{\prime}$ and $R$ have the same number of components, so each component of $R$ provides roles for exactly one component of $G^{\prime}$. It is impossible to make a role assignment from $\tilde{C}_{i}$ to $C_{j}$ when $\left|V_{\tilde{C}_{i}}\right|<\left|V_{C_{j}}\right|$. Hence the component $G$ can only be assigned roles of one of the components of maximum size.

If the roles of the component $G$ belong to $C_{m}$, then we are finished. Suppose the roles of the component $G$ are in $C_{i}$ with $i \neq m$. Then $\tilde{C}_{i}$ maps to some other component $C_{j}$ such that $\left|V_{\tilde{C}_{i}}\right|=\left|V_{C_{j}}\right|$ and by Observation 2.6 we get $\tilde{C}_{i} \simeq C_{j}$. 
Then $G$ allows also a $C_{j}$-role assignment. If $j \neq m$ we repeat the argument, and after at most $m$ iterations we find a desired $C_{m}$-role assignment of $G$.

Now we show that without the condition of global surjectivity " $r\left(V_{G}\right)=V_{R}$ ", some polynomially solvable $\mathrm{RA}(R)$ problems exist for role graphs $R$ with large components.

Take any role graph $R$ with bipartite components (of arbitrary size) but assure that at least one of these components is isomorphic to $K_{2}$ (i.e. to a graph consisting of two vertices forming an edge). For simplicity assume that $R$ has no isolated vertices. We claim that $G$ is $R$-role assignable if and only if $G$ is bipartite without isolated vertices. The necessity of such condition follows from the fact that non-bipartite graphs have no homomorphism to bipartite graphs. In the opposite direction, any homomorphism from $G$ to $K_{2}$ can be viewed as an $R$-role assignment of $G$.

This observation leads us to propose the following conjecture:

Conjecture For a simple role graph $R$, the $\mathrm{RA}(R)$ problem is solvable in polynomial time if and only if either all components of $R$ have at most two vertices, or $R$ is bipartite and at least one component is isomorphic to $K_{2}$. In all other cases the $\mathrm{RA}(R)$ problem is NP-complete.

Although we have shown above a proof of the polynomial part of the statement, we do not see a direct way for a possible NP-hardness construction.

\section{$6 \quad k$-Role Assignability}

In this section we study a more general version of the role assignment problem. We call a graph $G k$-role assignable if there exists a role graph $R$ on $k$ vertices, such that $G$ is globally $R$-role assignable.

$k$-Role Assignment ( $k$-RA)

Instance: A graph $G$.

Question: Is $G$ k-role assignable?

This problem was studied by [15] and is of interest in social network theory where networks are modeled in which individuals of the same social role relate to other individuals in the same way. The networks of individuals are represented by simple graphs. Contrary to our previous results, in this new model two individuals that are related to each other may have the same role. Hence role graphs that contain loops are allowed.

Again our aim is to fully characterize the computational complexity of the $k$-RA problem. Clearly the 1-RA problem is solvable in linear time, since it is sufficient to check whether $G$ has no edges $\left(R=K_{1}\right)$ or whether all vertices in $G$ have degree at least one ( $R$ consists of one vertex with a loop). The $2-$ $\mathrm{RA}$ problem is proven to be NP-complete in [15]. We generalize this result as follows:

Corollary 3 The $k$-RA problem is polynomially solvable for $k=1$ and it is NP-complete for all $k \geq 2$. 
Proof: We show that $k$-RA is NP-complete for $k \geq 3$. First assume that $k \geq 3$ but $k \neq 4$. We prove NP-completeness by reduction from $\mathrm{RA}\left(P_{k}\right)$, where $P_{k}$ is a path on $k$ vertices.

Let $G$ be an instance of $\operatorname{RA}\left(P_{k}\right)$ constructed in the proof of Theorem 1 . Without loss of generality we may assume that $G$ is connected and that a graph $\tilde{P}_{k} \simeq P_{k}$ is glued in $G$ by vertex $\tilde{v}$, the isomorphic copy of one of the two leaves of $P_{k}$. Let $G^{\prime}$ be the graph obtained after linking a path $P^{\prime}$ on $2 k-2$ vertices to $G$ via an edge from $\tilde{v}$ to one of the leaves of $P^{\prime}$. Our claim is that $G$ is $P_{k}$-role assignable if and only if $G^{\prime}$ is $k$-role assignable.

Clearly, if $G$ is $P_{k}$-role assignable, then $G^{\prime}$ is $k$-role assignable.

In the opposite direction, consider any $k$-role assignment of $G$ with a connected role graph $R$ on $k$ vertices. Denote vertices of $\tilde{P}_{k}$ by $\left\{\tilde{v}=u_{1}, u_{2}, \ldots, u_{k}\right\}$. Since $u_{k}$ is a leaf, it must be mapped to a leaf, and then by downward induction each $u_{i}: 2 \leq i<k$ has neighbors of two distinct roles. Otherwise $R$ cannot be connected and hence cannot be used for a global $R$-role assignment of $G$.

From the above we conclude that $R$ must be isomorphic to $P_{k}$ or otherwise to a path on $k$ vertices with a loop in one of its end points. However, the latter case leads to a contradiction if we try to assign roles to $P^{\prime}$. Hence $G^{\prime}$ can only be $P_{k}$-role assignable if it is $k$-role assignable. Clearly, this implies that $G$ is $P_{k}$-role assignable as well.

When $k=4$ we can either adjust the NP-completeness proof for $\mathrm{RA}\left(P_{4}\right)$ from [14] or reduce the $P_{3}$-role assignment problem by extending a graph $G^{\prime}$ obtained from a connected instance $G$ of $\mathrm{RA}\left(P_{3}\right)$ by an extra isolated vertex $u$. Then, by the same arguments we get that the original $G$ has to have an $P_{3}$-role assignment. This is because exactly one isolated vertex must be reserved as a role for $u$ in a possible $R$. (In fact $R$ is isomorphic to a disjoint union of $P_{3}$ and $K_{1}$.)

\section{Conclusion}

We have fully characterized the computational complexity for the $R$-role assignment problem for simple connected role graphs $R$. We have provided further arguments for disconnected role graphs too.

The computational complexity of the role assignment problem can be studied also for role graphs that contain some loops. If all components of $R$ either consist of exactly one vertex or are isomorphic to $K_{2}$, the $\mathrm{RA}(R)$ problem is polynomially solvable. The conjecture is that in all other cases the problem is NP-complete, even if instances are restricted to simple graphs.

We expect that our constructions would work in a similar way. Instead of a graph isomorphic to the role graph an other appropriate graph should be glued in the instance graph to obtain a reduction from the $\mathrm{H} 2 \mathrm{C}$ problem as we have used in the proof of Theorem 1.

Acknowledgements. The second author thanks Gerhard J. Woeginger for introducing the problem to him and for fruitful discussions on the subject. Both authors thank 
Petter Kristiansen and Jan Arne Telle for interesting comments on the NP-hardness construction.

\section{References}

[1] Abello, J., Fellows, M. R., And Stillwell, J. C. On the complexity and combinatorics of covering finite complexes. Australian Journal of Combinatorics 4 (1991), 103-112.

[2] Angluin, D. Local and global properties in networks of processors. In Proceedings of the 12th ACM Symposium on Theory of Computing (1980), 82-93.

[3] Angluin, D., And Gardiner, A. Finite common coverings of pairs of regular graphs. Journal of Combinatorial Theory B 30 (1981), 184-187.

[4] Biggs, N. Constructing 5-arc transitive cubic graphs. Journal of London Mathematical Society II. 26 (1982), 193-200.

[5] Bodlaender, H. L. The classification of coverings of processor networks. Journal of Parallel Distributed Computing 6 (1989), 166-182.

[6] Everett, M. G., And Borgatti, S. Role colouring a graph. Mathematical Social Sciences 21, 2 (1991), 183-188.

[7] Fiala, J., Heggernes, P., Kristiansen, P., and Telle, J. A. Generalized $h$-coloring and $h$-covering of trees. accepted to WG 2002.

[8] Fiala, J., And Kratochvíl, J. Complexity of partial covers of graphs. In Algorithms and Computation, 12th ISAAC'01, Christchurch, New Zealand (2001), no. 2223 in Lecture Notes in Computer Science, Springer Verlag, pp. $537-549$.

[9] Fiala, J., And Kratochvíl, J. Partial covers of graphs. Discussiones Mathematicae Graph Theory 22 (2002), 89-99.

[10] Fiala, J., Kratochvíl, J., And Kloks, T. Fixed-parameter complexity of $\lambda$-labelings. Discrete Applied Mathematics 113, 1 (2001), 59-72.

[11] Garey, M. R., And Johnson, D. S. Computers and Intractability. W. H. Freeman and Co., New York, 1979.

[12] Heggernes, P., And Telle, J. A. Partitioning graphs into generalized dominating sets. Nordic Journal of Computing 5, 2 (1998), 128-142.

[13] Kratochvíl, J., Proskurowski, A., And Telle, J. A. Covering regular graphs. Journal of Combinatorial Theory B 71, 1 (1997), 1-16. 
[14] Kristiansen, P., And Telle, J. A. Generalized $H$-coloring of graphs. In Algorithms and Computation, 11th ISAAC '01, Taipei, Taiwan (2000), no. 1969 in Lecture Notes in Computer Science, Springer Verlag, pp. 456466.

[15] Roberts, F. S., And Sheng, L. How hard is it to determine if a graph has a 2-role assignment? Networks 37, 2 (2001), 67-73. 